WU B 95-35

hep-ph/9503289

\title{
MODELLING THE NUCLEON WAVE FUNCTION FROM SOFT AND HARD PROCESSES
}

\author{
J. BOLZ ${ }^{* \dagger}$ and P. KROLL ${ }^{\ddagger}$ \\ Fachbereich Physik, Universität Wuppertal \\ D-42097 Wuppertal, Germany
}

(May 10, 2018)

\footnotetext{
*E-mail address: bolz@wpts0.physik.uni-wuppertal.de

${ }^{\ddagger}$ E-mail address: kroll@wpts0.physik.uni-wuppertal.de

${ }^{\dagger}$ Supported by the Deutsche Forschungsgemeinschaft
} 


\begin{abstract}
Current light-cone wave functions for the nucleon are unsatisfactory since they are in conflict with the data of the nucleon's Dirac form factor at large momentum transfer. Therefore, we attempt a determination of a new wave function respecting theoretical ideas on its parameterization and satisfying the following constraints: It should provide a soft Feynman contribution to the proton's form factor in agreement with data; it should be consistent with current parameterizations of the valence quark distribution functions and lastly it should provide an acceptable value for the $J / \psi \rightarrow N \bar{N}$ decay width. The latter process is calculated within the modified perturbative approach to hard exclusive reactions. A simultaneous fit to the three sets of data leads to a wave function whose $x$-dependent part, the distribution amplitude, shows the same type of asymmetry as those distribution amplitudes constrained by QCD sum rules. The asymmetry is however much more moderate as in those amplitudes. Our distribution amplitude resembles the asymptotic one in shape but the position of the maximum is somewhat shifted.
\end{abstract}

12.38.Bx,13.25.Gv, 13.40.Gp, 14.20.Dh

Typeset using REVTEX 


\section{INTRODUCTION}

There is general agreement that the conventional hard scattering approach (see [1] and references cited therein) in which the collinear approximation is used, gives the correct description of electromagnetic form factors and perhaps of other exclusive processes in the limit of asymptotically high momentum transfers. This framework relies upon the factorization of hadronic amplitudes in perturbative, short-distance dominated hard scattering amplitudes and process-independent soft distribution amplitudes (DA). In order to challenge arguments against the applicability of the hard scattering approach in experimentally accessible regions of momentum transfer [2] a modification of this scheme has been proposed by Botts, Li and Sterman [3] in which the transverse hadronic structure is retained and gluonic radiative corrections in form of a Sudakov factor are incorporated. This more refined treatment of exclusive observables allows to calculate the genuinely perturbative contribution self-consistently in the sense that the bulk of the perturbative contribution is accumulated in regions of reasonably small values of the strong coupling constant $\alpha_{S}$. Nevertheless it turns out that the perturbative contributions to exclusive observables are in general too small with perhaps a few exceptions. In particular in the case of the nucleon's Dirac form factor it is shown in Ref. [4] and is further confirmed in Ref. [5] that the perturbative contribution to it is indeed much smaller than the experimental data. For a plausible value of the mean transverse momentum inside the nucleon the perturbative contribution to the proton form factor amounts to less than about $10 \%$ of the experimental value 4,5 .

Ostensible agreement between data and calculations carried through within the conventional hard scattering approach under use of end-point concentrated DAs like that one proposed by Chernyak et al. (COZ) [6], can be traced back to large contributions from the soft end-point regions where one of the momentum fractions $x_{i}$ tends to zero. In these regions gluon momenta become small and hence the use of perturbation theory is unjustified [2]. 
The smallness of the perturbative contribution is, as we believe, not a debacle. On the contrary it seems to be fully consistent within the entire approach. We remind the reader of the contribution from the overlap of the initial and final state (soft) nucleon wave functions. This additional contribution, customarily termed the Feynman contribution, is usually neglected but it is indeed large, as was shown in Ref. [2] for a number of examples and as we are going to demonstrate for a wide class of wave functions. For the end-point concentrated wave functions like those based on the COZ DA [6], the Feynman contributions even exceed the experimental data [7] on the Dirac form factor of the nucleon, $F_{1}^{N}$, by large amounts. This parallels observations made on the pion's electromagnetic form factor recently (see, for instance, [ [ ] ) according to which end-point concentrated pion wave functions are clearly disfavoured.

The purpose of the present paper is the construction of the nucleon's (valence Fock state) wave function. In accord with the findings in [4,5] we demand this wave function to provide a Feynman contribution that completely controls the Dirac form factor at momentum transfers around $10 \mathrm{GeV}^{2}$. Admittedly, this requirement does not suffice to determine the wave function, further constraints are required. So we use the available information on the parton distribution functions [9] to which the nucleon's wave function is also related. As a third constraint we employ the decay reaction $J / \Psi \rightarrow N \bar{N}$. This process is expected to be dominated by perturbative contributions. We are going to calculate the decay width for it within the modified perturbative approach of Ref. [3] in contrast to previous analyses of the $J / \Psi$ decay [10 13]. Employing a parameterization of the wave function that complies with theoretical ideas [14, 15], we determine the few (actually two) parameters of the wave function from a combined fit to the data of the three reactions just mentioned. We emphasize that we do not aim at a perfect fit to the data, a number of theoretical uncertainties and approximations inherent in our approach would render such an attempt meaningless. The purpose of our analysis is rather to demonstrate the existence of a reasonable wave function from which the prominent features of the data can be reproduced. 
The paper is organized as follows: In Sect. II we briefly recapitulate a few properties of the nucleon's light-cone wave function and we introduce our ansatz for it. Sects. III, IV and $\mathrm{V}$ are devoted to the discussions of the Feynman contributions to $F_{1}^{N}$, the parton distribution functions and the decay $J / \psi \rightarrow N \bar{N}$, respectively. In Sect. VI we will present a new wave function satisfying the constraints discussed in Sects. III, IV and V. Finally, Sect. VII contains our conclusions. An appendix includes a derivation of the nucleonic $J / \psi$ width within the modified perturbative approach.

\section{THE NUCLEON WAVE FUNCTION}

Similarly to Sotiropoulos and Sterman [16] we write the valence Fock state of a proton with positive helicity as (the plane waves are omitted for convenience)

$$
\begin{aligned}
|P,+\rangle=\frac{1}{\sqrt{3 !}} \varepsilon_{a_{1} a_{2} a_{3}} \int[\mathrm{d} x]\left[\mathrm{d}^{2} \mathbf{k}_{\perp}\right]\{ & \Psi_{123} \mathcal{M}_{+-+}^{a_{1} a_{2} a_{3}}+\Psi_{213} \mathcal{M}_{-++}^{a_{1} a_{2} a_{3}} \\
& \left.-\left(\Psi_{132}+\Psi_{231}\right) \mathcal{M}_{++-}^{a_{1} a_{2} a_{3}}\right\}
\end{aligned}
$$

where we assume the proton to be moving rapidly in the 3-direction. Hence the ratio of transverse to longitudinal momenta of the quarks is small and one may still use a spinor basis on the light cone. A neutron state is obtained by the replacement $u \leftrightarrow d$. The integration measures are defined by

$$
[\mathrm{d} x]=\prod_{i=1}^{3} \mathrm{~d} x_{i} \delta\left(1-\sum_{i} x_{i}\right) \quad\left[\mathrm{d}^{2} \mathbf{k}_{\perp}\right] \equiv \frac{1}{\left(16 \pi^{3}\right)^{2}} \prod_{i=1}^{3} \mathrm{~d}^{2} \mathbf{k}_{\perp i} \delta^{(2)}\left(\sum_{i} \mathbf{k}_{\perp i}\right) .
$$

The quark $i$ is characterized by the usual fraction $x_{i}$ of the nucleon's momentum it carries,

by its transverse momentum $\mathbf{k}_{\perp i}$ with respect to the nucleon's momentum as well as by its helicity and color. A three-quark state is then given by

$$
\mathcal{M}_{\lambda_{1} \lambda_{2} \lambda_{3}}^{a_{1} a_{2} a_{3}}=\frac{1}{\sqrt{x_{1} x_{2} x_{3}}}\left|u_{a_{1}} ; x_{1}, \mathbf{k}_{\perp 1}, \lambda_{1}\right\rangle\left|u_{a_{2}} ; x_{2}, \mathbf{k}_{\perp 2}, \lambda_{2}\right\rangle\left|d_{a_{3}} ; x_{3}, \mathbf{k}_{\perp 3}, \lambda_{3}\right\rangle
$$

The quark states are normalized as follows

$$
\left\langle q_{a_{i}^{\prime}} ; x_{i}^{\prime}, \mathbf{k}_{\perp i}^{\prime}, \lambda_{i}^{\prime} \mid q_{a_{i}} ; x_{i}, \mathbf{k}_{\perp i}, \lambda_{i}\right\rangle=2 x_{i}(2 \pi)^{3} \delta_{a_{i}^{\prime} a_{i}} \delta_{\lambda_{i}^{\prime} \lambda_{i}} \delta\left(x_{i}^{\prime}-x_{i}\right) \delta\left(\mathbf{k}_{\perp i}^{\prime}-\mathbf{k}_{\perp i}\right) .
$$


Since the 3-component of the orbital angular momentum $L_{z}$ is assumed to be zero the quark helicities sum up to the nucleon's helicity. As has been demonstrated explicitly in Ref. [17 (2.1) is the most general ansatz for the $L_{z}=0$ projection of the three-quark nucleon wave function: From the permutation symmetry between the two $u$ quarks and from the requirement that the three quarks have to be coupled in an isospin $1 / 2$ state it follows that there is only one independent scalar wave function which, for convenience, we write as 4

$$
\Psi_{123}\left(x, \mathbf{k}_{\perp}\right) \equiv \Psi\left(x_{1}, x_{2}, x_{3} ; \mathbf{k}_{\perp 1}, \mathbf{k}_{\perp 2}, \mathbf{k}_{\perp 3}\right)=\frac{1}{8 \sqrt{3 !}} f_{N}\left(\mu_{F}\right) \phi_{123}\left(x, \mu_{F}\right) \Omega\left(x, \mathbf{k}_{\perp}\right)
$$

$f_{N}\left(\mu_{F}\right)$ plays the rôle of the nucleon wave function at the origin of the configuration space and the factorization scale is denoted by $\mu_{F} . \phi_{i j k}\left(x, \mu_{F}\right) \equiv \phi\left(x_{i}, x_{j}, x_{k}, \mu_{F}\right)$ is the nucleon DA conventionally normalized to unity

$$
\int[\mathrm{d} x] \phi_{123}\left(x, \mu_{F}\right)=1
$$

The DA is commonly expanded in a series of eigenfunctions $\tilde{\phi}_{123}^{n}(x)$ of the evolution kernel being linear combinations of Appell polynomials (see Ref. [1]

$$
\phi_{123}\left(x, \mu_{F}\right)=\phi_{\mathrm{AS}}(x)\left[1+\sum_{n=1}^{\infty} B_{n}\left(\mu_{F}\right) \tilde{\phi}_{123}^{n}(x)\right]
$$

where $\phi_{\mathrm{AS}}(x) \equiv 120 x_{1} x_{2} x_{3}$ is the asymptotic (AS) DA [1]. Evolution is incorporated by the scale dependences of $f_{N}$ and the expansion coefficients $B_{n}$ :

$f_{N}\left(\mu_{F}\right)=f_{N}\left(\mu_{0}\right)\left(\frac{\ln \left(\mu_{0} / \Lambda_{\mathrm{QCD}}\right)}{\ln \left(\mu_{F} / \Lambda_{\mathrm{QCD}}\right)}\right)^{2 / 3 \beta_{0}} \quad B_{n}\left(\mu_{F}\right)=B_{n}\left(\mu_{0}\right)\left(\frac{\ln \left(\mu_{0} / \Lambda_{\mathrm{QCD}}\right)}{\ln \left(\mu_{F} / \Lambda_{\mathrm{QCD}}\right)}\right)^{\tilde{\gamma}_{n} / \beta_{0}}$

where $\beta_{0} \equiv 11-2 / 3 n_{f}$ and $\mu_{0}$ denotes the scale of reference customarily chosen to be $1 \mathrm{GeV}$. The exponents $\tilde{\gamma}_{n}$ are the reduced anomalous dimensions. Because they are

\footnotetext{
${ }^{1}$ In [17] it has been shown that the entire nucleon state (including the $L_{z} \neq 0$ projections) is described by three independent functions.

${ }^{2}$ Note that the $\tilde{\phi}_{123}^{2}(x)$ used here differs from that of Ref. [1] by an overall sign.
} 
positive fractional numbers increasing with $n$ [18, higher order terms in (2.7) are gradually suppressed.

Nucleon DAs are frequently utilized in applications of the perturbative approach which are constrained by moments of the DA

$$
\phi^{\left(n_{1} n_{2} n_{3}\right)}\left(\mu_{0}\right)=\int[\mathrm{d} x] x_{1}^{n_{1}} x_{2}^{n_{2}} x_{3}^{n_{3}} \phi_{123}\left(x, \mu_{0}\right)
$$

evaluated by means of QCD sum rules [6]. The few moments known only suffice to determine the first five expansion coefficients $B_{n}$. However, since the moments are burdened by errors the $B_{n}$, and hence the DA, are not fixed uniquely. In Ref. [19], for instance, a set of 45 model DAs has been constructed where each DA respects the moments of Ref. [6] and is strongly end-point concentrated and asymmetric in the $x_{i}$. It is shown in Ref. [4], as we already mentioned in the introduction, that the perturbative contributions to the nucleon form factor evaluated with these DAs are too small in comparison with the data [7]. As we are going to discuss subsequently the DAs constructed by Bergmann and Stefanis [19] also show serious deficiencies in other applications. The value of $f_{N}$ is also determined from QCD sum rules [6]: $(5.0 \pm 0.3) \cdot 10^{-3} \mathrm{GeV}^{2}$. This value is to be used in conjunction with the COZ DA [6] and the DAs of Ref. [19].

The transverse momentum dependence of the wave function is contained in the function $\Omega$ which is normalized according to

$$
\int\left[\mathrm{d}^{2} \mathbf{k}_{\perp}\right] \Omega\left(x, \mathbf{k}_{\perp}\right)=1
$$

Throughout we use a simple symmetric Gaussian parameterization for the $k_{\perp}$-dependence

$$
\Omega\left(x, \mathbf{k}_{\perp}\right)=\left(16 \pi^{2}\right)^{2} \frac{a^{4}}{x_{1} x_{2} x_{3}} \exp \left[-a^{2} \sum_{i=1}^{3} k_{\perp i}^{2} / x_{i}\right]
$$

which resembles the harmonic oscillator wave function proposed in [14]. The ansatz (2.11) keeps our model simple and appears to be reasonable for a nucleon wave function $\Psi_{123}$ which is dominantly symmetric. With the ansatz (2.7), (2.11) antisymmetric or mixed symmetric contributions may only appear through the DA. 
For reasons which will become clear subsequently we only need the soft part of the wave function, i.e. the full wave function with its perturbative tail removed from it [1]. The Gaussian $k_{\perp}$-dependence is conform with the behaviour of a soft wave function; the powerlike decreasing perturbative tail is removed. The ansatz (2.11) is also supported by recent work of Chibisov and Zhitnitsky [15] who showed that, on rather general grounds, $\Omega$ depends on $x_{i}$ and $\mathbf{k}_{\perp i}$ solely in the combination $k_{\perp i}^{2} / x_{i}$ and that $\Omega$ falls off like a Gaussian at large $k_{\perp}$. . $^{2}$ Eq. (2.11) is the simplest way to comply with these requirements. We remark that integrating $\Omega$ in Eq. (2.10) to infinity instead of to a cut-off scale of order $Q$ introduces only a small negligible error into the calculation.

According to (2.5) a wave function is defined by a certain DA combined with the Gaussian (2.11) and $f_{N}$. For the COZ wave function which we will subsequently confront to data for the purpose of comparison, the transverse size parameter $a$, controlling the width of the wave function in $k_{\perp}$ space, is fixed by requiring a certain value of either the root mean square (rms) transverse momentum or the probability of the valence Fock state. We will utilize the COZ wave function for three cases: $P_{3 q}=1\left(a=0.99 \mathrm{GeV}^{-1},\left\langle k_{\perp}^{2}\right\rangle^{1 / 2}=272\right.$ $\mathrm{MeV}),\left\langle k_{\perp}^{2}\right\rangle^{1 / 2}=450 \mathrm{MeV}\left(a=0.60 \mathrm{GeV}^{-1}, P_{3 q}=0.13\right)$ and $600 \mathrm{MeV}\left(a=0.45 \mathrm{GeV}^{-1}\right.$, $\left.P_{3 q}=0.04\right)$.

\section{THE FEYNMAN CONTRIBUTION}

The Dirac form factor of the nucleon can be expressed in terms of overlaps of initial and final Fock state wave functions [20,21]. This is an exact representation of the form factor provided a sum over all Fock states is implied and the full Fock state wave functions

\footnotetext{
${ }^{3}$ The kinematical transverse momentum of the partons is not the same object as $k_{\perp}$ defined through moments as in [15]. However, we will assume that both are one and the same variable. This assumption corresponds to summing up soft gluon corrections, i.e. higher twist contributions.
} 
are used. However, one can identify the overlaps of the hard large $k_{\perp}$ tails of the wave functions with the perturbative contributions [1]. Since this contribution is small [4] the form factor is dominated by the overlaps of the soft parts of the wave functions. The physical picture behind the overlap representation is that one single quark is scattered by the virtual photon with the remaining constituents following the struck quark as spectators. The various overlap integrals are dominated by those configurations where the struck quark carries almost the entire momentum of the nucleon. Obviously, with an increasing number of partons sharing the nucleon's momentum it becomes less likely that one parton carries the full momentum of the nucleon. Therefore, higher Fock state contributions are strongly suppressed at large momentum transfer. The valence Fock state provides the most important soft contribution (this is termed the Feynman contribution) in the region of momentum transfer around $10 \mathrm{GeV}^{2}$. Of course, as required by the consistency of the entire picture, the perturbative contribution will take control in the limit $Q \rightarrow \infty$; the Feynman contribution is suppressed by powers of $Q$ relative to the perturbative contribution.

According to Drell and Yan [20] we calculate the nucleon matrix elements of the electromagnetic current in a frame where the incoming nucleon is rapidly moving in the 3-direction (infinite momentum frame). To leading order in the nucleon's momentum $P$ we find from (2.1), combined with the ansatz (2.5) for the wave function, the following expression for the Feynman contribution to the Dirac form factor

$$
\begin{aligned}
F_{1 \text { soft }}^{N}\left(Q^{2}\right) & =\left(\frac{f_{N}}{8 \sqrt{6}}\right)^{2} \sum_{j=1}^{3} e_{j} \int[\mathrm{d} x]\left[\phi_{123}^{2}(x)+\phi_{213}^{2}(x)+\left(\phi_{132}(x)+\phi_{231}(x)\right)^{2}\right] \\
& \times \int\left[\mathrm{d}^{2} \mathbf{k}_{\perp}\right] \Omega\left(x, \mathbf{k}_{\perp j}+\left(1-x_{j}\right) \mathbf{q}, \mathbf{k}_{\perp i}-x_{i} \mathbf{q}\right) \Omega\left(x, \mathbf{k}_{\perp}\right) .
\end{aligned}
$$

This result is obtained from the matrix elements of the so-called good current components $(\mu=0,3)$. For $P \rightarrow \infty$ these matrix elements are only fed by such configurations for which all constituents of the nucleon move along the same direction as the nucleon (up to finite transverse momenta), i.e. $0 \leq x_{i} \leq 1$ for all constituents [22]. Matrix elements of the bad current components $(\mu=1,2)$ have to be treated with precaution; they are 
less reliable. Indeed, these matrix elements are suppressed by $1 / P$ as opposed to those of the good current components and suffer from many approximations made, e.g. off-shell effects, helicity or spin rotations and so on. Therefore, we also refrain from calculating the Pauli form factor, $F_{2}$, along the same lines as $F_{1}$ since it is controlled by such non-leading (with respect to the momentum $P$ ) contributions. To be more specific, $F_{2}$ can only be calculated if $L_{z} \neq 0$ components of the nucleon wave function are also included. We also note that the expression (3.1) somewhat differs from overlap expressions given by Isgur and Llewellyn-Smith [2] who start from current matrix elements in a Breit frame and boost them to the infinite momentum frame. In this procedure spin rotations have to be considered which, in a model dependent way, generate $L_{z} \neq 0$ components in the nucleon wave function. Still the numerical results obtained in Ref. [2] are very similar to our ones. For a completely symmetric wave function the expression (3.1) is proportional to the sum of the quark charges and thus exactly vanishes in the case of the neutron form factor $F_{1}^{n}$. I This observation already precludes the AS wave function. For this reason we refrain from showing results on $F_{1 \text { soft }}^{p}$ evaluated with the AS wave function. The integrations in (3.1) can easily be performed analytically for wave functions of the type we consider consisting of the Gaussian (2.11) and a DA for which the expansion (2.7) is truncated at some finite $n$. For such wave functions the Feynman contribution falls off proportional to $Q^{-8}$ for $Q^{2} \rightarrow \infty$

In Fig. 1 we show the Feynman contribution to the proton form factor evaluated with the

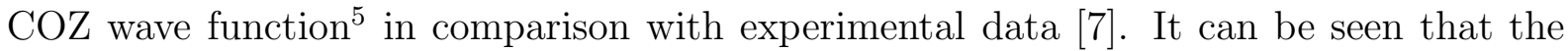
data is dramatically exceeded by the Feynman contribution. This result is independent on the value of the transverse size parameter used; an increase of the $k_{\perp}$-width only shifts

\footnotetext{
${ }^{4}$ The neutron form factor is zero for any wave function at zero momentum transfer.

${ }^{5}$ As the external $Q^{2}$ is the only scale present in the process it defines at the same time a natural evolution scale for the DA.
} 
the position of the maximum value of the Feynman contribution to higher $Q^{2}$ without considerably reducing its magnitude. The asymptotic $Q^{-8}$ behaviour does not set in before $Q^{2} \approx 100 \mathrm{GeV}^{2}$ (since the expansion of (3.1) into a series of $1 / Q^{2}$ powers converges slowly). Similarly large Feynman contributions are obtained from the COZ wave function in the case of the neutron.

The COZ wave function is representative for all strongly end-point concentrated DAs. We checked that all wave functions constructed from the Bergmann-Stefanis set of DAs [19] provide similar unphysically strong Feynman contributions as the COZ wave function [5]. As was found in [2,23] an $x$-independent Gaussian instead of our ansatz (2.11), also leads to large Feynman contributions to $F_{1}$ for the COZ DA. These findings give rise to severe objections against DAs constrained by the QCD sum rule moments of Ref. [6].

The Feynman contributions are also subject to Sudakov corrections. An estimate of these corrections on the basis of the Sudakov factor as derived by Botts and Sterman [3] (see (A7)) reveals that the size of the Feynman contributions is somewhat reduced by it. The suppression is stronger for the end-point concentrated wave functions (but the Feynman contributions still exceed the form factor data dramatically) than for the AS wave function or similar ones. Since this suppression can be compensated for by readjusting the wave function parameters we refrain from taking into account the Sudakov corrections to the Feynman contributions.

\section{VALENCE QUARK DISTRIBUTION FUNCTIONS}

Deep inelastic lepton-nucleon scattering provides information on the parton distributions inside nucleons. As is discussed in [14] the parton distribution functions $q^{N}(x)$ representing the number of partons of type $q$ with momentum fraction $x$ inside the nucleon, are determined by the Fock state wave functions. Each Fock state contributes through the modulus squared of its wave function, integrated over transverse momenta

up to $Q$ and over all fractions except those pertaining to partons of type $q$. Obviously, 
the valence Fock state wave function only feeds the valence quark distribution functions, $u_{V}^{N}(x)$ and $d_{V}^{N}(x)$. Since each Fock state contributes positively the following inequalities hold

$$
\begin{aligned}
& u_{V}^{p}(x) \geq 2 \int[\mathrm{d} x]\left[\mathrm{d}^{2} \mathbf{k}_{\perp}\right]\left[\Psi_{123}^{2}+\Psi_{213}^{2}+\left(\Psi_{132}+\Psi_{231}\right)^{2}\right] \delta\left(x-x_{1}\right), \\
& d_{V}^{p}(x) \geq \int[\mathrm{d} x]\left[\mathrm{d}^{2} \mathbf{k}_{\perp}\right]\left[\Psi_{123}^{2}+\Psi_{213}^{2}+\left(\Psi_{132}+\Psi_{231}\right)^{2}\right] \delta\left(x-x_{3}\right) .
\end{aligned}
$$

In (4.1) the full wave function enters. For reasons obvious from the preceding discussions, we evaluate the valence Fock state contributions to $u_{V}^{p}$ and $d_{V}^{p}$ from our soft wave functions. On the strength of our experience with the nucleon form factor we expect the neglect of the perturbative tails to be admissible. The soft wave functions defined by (2.5), (2.7) - with the expansion truncated at some finite order - and (2.11) leads to the behaviour $x q_{V}^{N}(x) \sim(1-x)^{3}$ for $x \rightarrow 1$ in fair agreement with the structure function data. This property is related to the asymptotic behaviour of the Feynman contribution $F_{1}^{N}$ soft $\sim Q^{-8}$. The interrelation between a $(1-x)^{p}$ behaviour of the distribution functions for $x \rightarrow 1$ and a $(1 / Q)^{p+1}$ behaviour of the Feynman contribution at large $Q^{2}$ proposed by Drell and Yan [20] is only obtained for wave functions factorizing in $x$ and $k_{\perp}$ (i.e. for $\Omega$ not depending on the $x_{i}$ ). Asymptotically, if the perturbative contribution dominates the form factor, the Drell-Yan interrelation also holds for wave functions of the type we consider.

Another interesting property of (4.1) is that any symmetric wave function, as e.g. the AS one, provides a value of 2 for the ratio $u_{V}^{p}(x) / d_{V}^{p}(x)$. This is to be contrasted with the value of 5 (at $x \approx 0.6$ ) for that ratio to be seen in current parameterizations of the distribution functions [9].

In Fig. 2 we compare the valence Fock state contributions, evaluated with the COZ wave function, to $u_{V}^{p}$ and $d_{V}^{p}$ with the Glück-Reya-Vogt parameterizations [9] at a scale of 1 $\mathrm{GeV}^{2}$ in the large $x$ region. For $x$ smaller than 0.6 our results well respect the inequalities (4.1). For $x$ larger than about 0.6 , on the other hand, wave functions normalized to large probabilities (or equivalently providing small values of the mean transverse momentum) 
violate the inequalities (4.1). For the COZ wave function consistency with the GlückReya-Vogt parameterizations can only be achieved if $P_{3 q}$ is less than about $1 \%$ which for our wave function parameterization would correspond to unrealistically large mean transverse momenta ( $\gtrsim 1 \mathrm{GeV}$ ). Similar results are found for the other COZ-like wave functions constructed from the Bergmann-Stefanis set of DAs [19]. This observation is to be considered as another serious failure of the COZ-like wave functions. It should be mentioned that Schäfer et al. [24] found similar results for the valence quark distribution functions or structure functions, respectively.

\section{V. $J / \psi$ DECAY INTO NUCLEON-ANTINUCLEON}

The decay width of the process $J / \psi \rightarrow N \bar{N}$ provides a third constraint on the nucleon wave function. By reason of the spin and the parity of the $J / \psi$ meson and of color neutralization the massive charm quarks the $J / \psi$ is composed of, dominantly annihilate through three gluons which in turn create the light quark pairs necessary for the formation of nucleon and antinucleon (see Fig. 3 ). There are hints that both the $c \bar{c}$ annihilation and the conversion of the gluons into $N \bar{N}$ pairs, are under control of perturbative QCD. If, for instance, the decays of charmonium states into light hadrons are viewed as decays into two or three gluons the widths can be estimated perturbatively. With acceptable values of $\alpha_{S}$ one obtains reasonable agreement with experiment [25] although in the case of the $J / \psi$ that value appears to be a bit small with regard to the relevant scale provided by the charm quark mass (see the discussion in [26]). Another hint that perturbative QCD is at work in $J / \psi$ decays is provided by the angular distribution of $N \bar{N}$ pairs produced via $e^{+} e^{-} \rightarrow J / \psi \rightarrow N \bar{N}$. From the data of the DM2 collaboration [27] one estimates the

fraction of $N \bar{N}$ pairs with equal helicities to amount to $(10 \pm 3) \%$ of the total number of pairs. This is what is to be expected if the process is dominated by perturbative QCD: Each of the virtual gluons produces a light, almost massless quark and a corresponding antiquark with opposite helicities. Since our nucleon wave function does not embody 
any non-zero orbital angular momentum component the quark helicities sum up to the nucleon's helicity. Hence, nucleon and antinucleon are dominantly produced with opposite helicities. This fact is an example of the well-known helicity conservation rule for light hadrons [1:10]. The small amount of $N \bar{N}$ pairs with the wrong helicity combination observed experimentally, while indicating the presence of some soft contributions, can be tolerated. One should, however, be aware of these contributions when theoretical results for the $J / \psi \rightarrow N \bar{N}$ decay are compared with experiment.

It is, however, fair to mention that there are several difficulties with the perturbative calculation of exclusive charmonium decays, e.g. the relatively large branching ratios of $\psi^{\prime}(2 s) \rightarrow p \bar{p}, \eta_{c} \rightarrow p \bar{p}$ and $J / \psi \rightarrow \pi \rho$. We will nevertheless calculate the $J / \psi \rightarrow$ $N \bar{N}$ decay width within a perturbative approach. It will turn out and this may be regarded as another argument in favour of the prominent rôle of perturbative QCD in charmonium decays, that the same wave function that, on the one hand, leads to a very small perturbative contribution to the nucleon form factor (and simultaneously to a large Feynman contribution), provides, on the other hand, a reasonably large value for the $J / \psi \rightarrow N \bar{N}$ decay width.

We start the calculation of the $J / \psi \rightarrow N \bar{N}$ decay width from an invariant decomposition of the decay helicity amplitudes (with an appropriately chosen spin quantization axis of the $J / \Psi)$

$$
\mathcal{M}_{\lambda_{1} \lambda_{2} \lambda}=\bar{u}\left(p_{1}, \lambda_{1}\right)\left[\mathcal{B}\left(M_{\psi}^{2}\right) \gamma_{\mu}+\mathcal{C}\left(M_{\psi}^{2}\right) \frac{\left(p_{1}-p_{2}\right)_{\mu}}{2 m_{N}}\right] v\left(p_{2}, \lambda_{2}\right) \epsilon^{\mu}(\lambda)
$$

where $p_{1}\left(p_{2}\right)$ and $\lambda_{1}\left(\lambda_{2}\right)$ are the momentum and the helicity of the nucleon (antinucleon) respectively. $u$ and $v$ denote the nucleon spinors and $\epsilon$ the polarization vector of the $J / \psi$. In the perturbative approach hadronic helicity conservation forces the invariant function $\mathcal{C}$ to vanish. The $J / \psi \rightarrow N \bar{N}$ decay width, therefore, takes the simple form (up to corrections of order $\left(m_{N} / M_{\psi}\right)^{4}$ where $M_{\psi}$ is the $J / \psi$ mass)

$$
\Gamma(J / \psi \rightarrow N \bar{N})=\frac{M_{\psi}}{12 \pi}|\mathcal{B}|^{2}
$$


There is a small, almost negligible contribution to the invariant function $\mathcal{B}$ from the $c \bar{c}$ annihilation mediated by two gluons and a photon. The $g g \gamma$ contribution to $\mathcal{B}$ being proportional to the $g g g$ contribution, amounts to about $1 \%$ of the latter. In the following it is understood that the $g g \gamma$ contribution is absorbed into the $g g g$ contribution. There is also a small electromagnetic contribution to the invariant function $\mathcal{B}$ from the $c \bar{c}$ annihilations through a virtual photon. This contribution involves the time-like Dirac and Pauli form factor of the nucleon

$$
\mathcal{B}_{\mathrm{em}}=\frac{8 \pi \alpha}{3 M_{\psi}} f_{\psi}\left(F_{1}^{N}\left(s=M_{\psi}^{2}\right)+\kappa_{N} F_{2}^{N}\left(s=M_{\psi}^{2}\right)\right)
$$

The factor multiplying the form factors is the invariant amplitude for the $J / \psi$ decay into a lepton pair $\left(m_{l}=0\right)$. This factor includes the $J / \psi$ decay constant $f_{\psi}$ (being related to the configuration space wave function at the origin) which can be determined from the experimental value of the leptonic decay widths [28]. One finds $f_{\psi}=409 \pm 14 \mathrm{MeV}$ from a leading order calculation. The $\alpha_{S}$ corrections to $f_{\Psi}$ are known to be large [29], leading to an increase of $f_{\Psi}$ by about $20 \%$. However, there is no assurance that the $\alpha_{S}^{2}$ corrections are not also large and perhaps cancelling partly the $\alpha_{S}$ correction. Therefore we use the leading order value of $f_{\Psi}$ being aware of this eventual source of uncertainty in our final result. Using $F_{1}^{p}+\kappa_{p} F_{2}^{p}=G_{M}^{p}=2.5 \pm 0.4 \mathrm{GeV}^{4} / M_{\psi}^{4}$ in agreement with the E760 data [30] and $F_{2}^{p}=0$, we estimate the electromagnetic contribution $\mathcal{B}_{\mathrm{em}}$ to the $J / \psi \rightarrow p \bar{p}$ invariant decay amplitude $\mathcal{B}$ to amount to $(14 \pm 4) \%$ of the experimental value.

Several QCD studies of the decay $J / \psi \rightarrow 3 g \rightarrow N \bar{N}$ have appeared in the past [10 13]. A point to criticize in these studies which relied on the conventional hard scattering approach, is the treatment of the strong coupling constant $\alpha_{S}$. Since, on the average, the virtuality of the intermediate gluons is roughly $1 \mathrm{GeV}^{2}$ one would expect $\alpha_{S}$ to be of the order of 0.4 to 0.5 rather than 0.2 to 0.3 which is usually chosen [11 13. Since $\alpha_{S}$ enters to the sixth power into the expression for the width a variation of $\alpha_{S}$ from, say, 0.3 to 0.45 would lead to a change by a factor of 11 for the width. Thus, a large uncertainty is hidden in these calculations preventing any severe test of the DA utilized. 
In constrast to previous work [10 13] we will not use the collinear approximation but rather use the modified perturbative approach of Sterman et al. [3] in which transverse degrees of freedom are retained and Sudakov suppression, comprising those gluonic radiative corrections not included in the evolution of the wave function, are taken into account. The calculation of the three-gluon contribution to the invariant function $\mathcal{B}$ is presented in some detail in Appendix A.

An important advantage of the modified perturbative approach is that the strong coupling constant can to be used in one-loop approximation; its singularity, to be reached in the end-point regions $x_{i} \rightarrow 0$, is compensated by the Sudakov factor. Hence, there is no uncertainty in its use. This is to be contrasted with the conventional perturbative approach where either $\alpha_{S}$ is evaluated at an $x$-independent renormalization scale typically chosen to be of the order of $M_{\psi}^{2}$ or at scales like (A4). In the latter case for which, in contrast with the first case, large logs from higher orders of perturbation theory are avoided, $\alpha_{S}$ has to be frozen in at a certain value (typically 0.5 ) in order to avoid uncompensated $\alpha_{S}$ singularities in the end-point regions. The modified perturbative approach possesses another interesting feature: the soft end-point regions are strongly suppressed. Therefore, the bulk of the perturbative contribution comes from regions where the internal quarks and gluons are far off-shell (order of $M_{\psi}^{2}$ ). In contrast to the nucleon form factor the $J / \psi \rightarrow N \bar{N}$ amplitude is not end-point sensitive. The suppression of the end-point regions does not, therefore, lead to a substantial reduction of the $J / \psi \rightarrow N \bar{N}$ amplitude.

As in Sects. III and IV and before we turn to the determination of a new nucleon wave function, we test the $\mathrm{AS}$ and the $\mathrm{COZ}$ wave functions. Evaluating the three-gluon contribution $\mathcal{B}_{3 g}$ from (A2) and leaving aside the inconsistencies with the form factor and

\footnotetext{
${ }^{6}$ This is due to the fact that in the collinear approximation the propagator denominators of the hard scattering amplitude vanish only linearly in the end-point regions whereas e.g. in the case of the nucleon form factor they vanish quadratically for some of the $x_{i}$.
} 
the valence quark distribution functions we find acceptable values for the decay width $\left(\Lambda_{\mathrm{QCD}}=220 \mathrm{MeV}\right):$ Using the AS wave function $\left(f_{N}=6.64 \cdot 10^{-3} \mathrm{GeV}^{2}, a=0.75 \mathrm{GeV}^{-1}\right)$ we find $\Gamma_{3 g}(J / \psi \rightarrow p \bar{p})=0.09 \mathrm{keV}$ and for the COZ wave function $\left(a=0.6 \mathrm{GeV}^{-1}\right) 0.23$ $\mathrm{keV}$. Smaller (higher) values for the width are obtained if the transverse size parameter $a$ is decreased (increased). For comparison the experimental value for the $J / \psi \rightarrow p \bar{p}$ decay width is $0.188 \pm 0.014 \mathrm{keV}$ [28]. Similar results for the width are obtained with the wave functions constructed from the Bergmann-Stefanis DAs [19. The only exception is the Gari-Stefanis DA [31] which provides a very small value for $\Gamma_{3 g}$ of the order of a few $\mathrm{eV}$.

\section{A MODEL FOR THE NUCLEON WAVE FUNCTION}

We have demonstated that the COZ-like wave functions as well as the AS one do not describe the three processes discussed in the preceding sections in a satisfactory manner. For this reason we will now try to determine a new wave function from a fit to the proton form factor data [7] (using (3.1)), the valence quark distribution functions of Glück et al. [9] (using (4.1)) as well as the $J / \psi \rightarrow p \bar{p}$ decay width 28] (using (A2)). We start from the ansatz (2.1), (2.5) and assume the Gaussian $k_{\perp}$-dependence (2.11) again. An important question is, how many terms one has to allow in the expansion (2.7). It would be obvious to truncate the expansion at $n=5$ again. As explorative fits immediately reveal, five terms in (2.7) provide too much freedom. For several expansion coefficients, in particular for $B_{5}$, one always obtains very small values. The corresponding terms in (2.7) can be neglected without worsening the fits noticeably. Indeed it suffices to consider

only the first order expansion terms, $\tilde{\phi}_{123}^{1}$ and $\tilde{\phi}_{123}^{2}$, and even that still implies unnecessary freedom in case both the coefficients, $B_{1}$ and $B_{2}$ are treated as free parameters. As it turns out ultimately, the simple DA

$$
\phi_{123}(x)=\phi_{\mathrm{AS}}(x)\left[1+\frac{3}{4} \tilde{\phi}_{123}^{1}(x)+\frac{1}{4} \tilde{\phi}_{123}^{2}(x)\right]=\phi_{\mathrm{AS}}(x) \frac{1}{2}\left[1+3 x_{1}\right]
$$


meets all requirements. The only free parameters left over in this case, namely $f_{N}$ and $a$, are determined by a fit to the data for the three processes mentioned above. The fit provides the following values for the two parameters: $f_{N}=6.64 \cdot 10^{-3} \mathrm{GeV}^{2}, a=0.75$ $\mathrm{GeV}^{-1}$. The fitted wave function implies a value of 0.17 for the probability of the valence Fock state and a value of $411 \mathrm{MeV}$ for the rms transverse momentum. Both values appear to be reasonable. We stress that a larger flexibility in the DA, i.e. allowing for more free parameters to be adjusted in the fit, does not improve the fit substantially. We also remark that the DA is not uniquely determined by the data. Another solution of similar quality exists for which the DA contains the expansion terms $\tilde{\phi}_{123}^{2}, \tilde{\phi}_{123}^{3}$ and $\tilde{\phi}_{123}^{4}$. Although the mathematical expression of that DA looks rather complicated the DA itself is similar to (6.1) in shape and magnitude.

Our value for $f_{N}$ is about $30 \%$ larger than that obtained from QCD sum rules [6]. In lattice QCD, on the other hand, the following values for $f_{N}$ are found: $(2.9 \pm 0.6) \cdot 10^{-3}$ $\mathrm{GeV}^{2}$ [32] and $6.6 \cdot 10^{-3} \mathrm{GeV}^{2}$ [33]. Thus, within a factor of about 2 all values agree with each other. With regards to the large systematic uncertainties in the various approaches the spread of the $f_{N}$ values cannot be considered as a contradiction.

The proton decay offers another check of our wave function. Calculating from it the three-quark annihilation matrix element of the proton (termed $\alpha$ in [34]) along the same lines as in [34], we find for it a value of $0.012 \mathrm{GeV}^{3}$ which is about three times smaller than the value quoted in Ref. [34]. Our value is almost identical to a recent result from lattice QCD [33] and rather close to many other results [35]. The source of the difference between our result and that one of Brodsky et al. [34] chiefly lies in the fact that we give up the idea of calculating the proton form factor perturbatively.

The DA (6.1), displayed in Fig. 4, possesses interesting features. It is much less asymmetric and less end-point concentrated than the COZ-like DAs which exhibit three pronounced maxima and regions where the DAs acquire negative values. Our DA rather resembles the AS one in shape but with the position of the only maximum shifted to $x_{1}=0.44$, $x_{2}=x_{3}=0.28$. Thus, as the COZ DA but to a lesser amount, our DA possesses the 
property that, on the average, a $u$-quark in the proton carries a larger fraction of the proton's momentum than the $d$-quark. Related to the shift of the maximum's position is the asymmetry in the first order moments of our DA:

$$
\left\langle x_{1}\right\rangle_{\mathrm{FIT}}=\frac{8}{21}, \quad\left\langle x_{2}\right\rangle_{\mathrm{FIT}}=\left\langle x_{3}\right\rangle_{\mathrm{FIT}}=\frac{13}{42},
$$

which values are to be contrasted with the QCD sum rule values [6]

$$
\left\langle x_{1}\right\rangle_{\mathrm{COZ}}=0.54-0.62, \quad\left\langle x_{2}\right\rangle_{\mathrm{COZ}}=0.18-0.20, \quad\left\langle x_{3}\right\rangle_{\mathrm{COZ}}=0.20-0.25 .
$$

The moments (6.2) are consistent with those obtained by Martinelli and Sachrajda from lattice QCD [32]. For a discussion of the various approximations made in the QCD sum rule analysis of [6, 36] and their implications see [37].

The DA (6.1) or, when combined with the totally symmetric Gaussian (2.11), the wave function, can be decomposed into a symmetric part and a part of mixed symmetry under permutations (we adopt the notations of Ref. [2])

$$
\phi^{S}(x)=-\sqrt{6} \phi_{A S}(x), \quad \phi^{\rho}(x)=-\frac{3}{2}\left(x_{1}-x_{2}\right) \phi_{A S}(x), \quad \phi^{\lambda}(x)=\frac{\sqrt{3}}{2}\left(1-3 x_{3}\right) \phi_{A S}(x) .
$$

These functions are related to the $\{56\}$ and $\{70, L=0\}$ representations of the permutation group on three objects. Representations with non-zero orbital angular momentum $L$ do not contribute to (6.1). The symmetric $\{56\}$ part is dominant since the ratio of probabilities $P_{3 q}^{S} / P_{3 q}$ is $28 / 29=0.9655$. The strength of the $\{70, L=0\}$ admixture is about the same as found from equal-time wave function analysis [38]. This observation provides some justification of the symmetric ansatz (2.11) for the $k_{\perp}$-dependence à posteriori.

The results for the valence quark distribution functions obtained from the fit are shown in Fig. 2. The valence Fock state contribution to $x d_{V}(x)$ comes out comparatively large leaving hardly room for contributions from higher Fock states for $x \gtrsim 0.6$. The effect of the asymmetric part of our DA provided by the eigenfunctions $\tilde{\phi}_{123}^{1}$ and $\tilde{\phi}_{123}^{2}$ is clearly visible in Fig. 2: It pushes up $u_{V}$ at large $x$ and diminishes $d_{V}$ at the same time, thus 
producing a ratio $u_{V}: d_{V}$ of about $5: 1$.

At this point it is in order to draw the reader's attention to a little difficulty: The evolution behaviour of our soft contributions to the valence quark distribution functions does not exactly match with that of the phenomenological distribution functions. This entails violations of the inequalities (4.1) for $Q^{2} \gg 1 \mathrm{GeV}^{2}$. The imperfect evolution behaviour appears as a consequence of several approximations made in our approach. Thus, for instance, we consider only the soft part of the wave function and yet extend the upper limit of the $\mathbf{k}_{\perp}$ integration to infinity (numerically this is of little importance for a Gaussian like (2.11) ). We also ignored a possible evolution of the transverse size parameter (see [14 where the pion case is discussed). With respect to our objective of a more qualitative understanding of the nucleon's form factors and distribution functions rather than a perfect quantitative description we tolerate that minor drawback.

In Fig. 5 we show the results for the Feynman contributions to the proton and neutron form factors in comparison with the data [7, 39]. While the data on $F_{1}^{p}$ is input to the fit the results for the neutron form factor are predictions. It can be seen that our wave function provides Feynman contributions which exhibit a broad maximum near $15 \mathrm{GeV}^{2}$. The asymmetric part of the DA (6.1) is solely responsible for the neutron form factor (pushing it down from zero to a negative value) and pushes up the Feynman contribution to the proton form factor (see the difference between the solid and dashed lines in Fig. 5). For $Q^{2}$ smaller than about $8 \mathrm{GeV}^{2}$ the fit is somewhat below the data. We are content with that result because, as we already mentioned, our goal is not a perfect fit but rather to demonstrate the existence of a wave function that provides a Feynman contribution to the nucleon's form factor of the right magnitude and that is consistent with the constraints from the other two processes we consider and thus implicitly gives an explanation for the smallness of the perturbative contribution to the nucleon's form factor. An improved fit in agreement with the form factor data over a wide range of $Q^{2}$ can likely be achieved with minor modifications of the Gaussian (2.11) at finite transverse momentum. Since such modifications, an example of which has been given by Zhitnitsky 40 for the case of 
the pion wave function, require more free parameters we persist in the simple Gaussian (6.1). For $Q^{2} \lesssim 8 \mathrm{GeV}^{2}$ the presence of higher Fock states is to be expected (remember $\left.P_{3 q}=0.17\right)$. The DA of a Fock state consisting of $n_{g}$ gluons and $n_{q}$ quarks contains to lowest order in the momentum fractions terms proportional to $x_{1} x_{2} \ldots x_{n_{q}} x_{n_{q}+1}^{2} \ldots x_{n_{q}+n_{g}}^{2}$ as is supported by power counting arguments given in [36]. Using this asymptotic form of a higher Fock state DA in combination with a Gaussian $k_{\perp}$-dependence of the type (2.11) one finds the Feynman contribution to fall off as $Q^{-4\left(n_{q}+2 n_{g}-1\right)}$. Hence, for this type of wave functions contributions from higher Fock states become strongly suppressed at large $Q^{2}$ but may be quite important below $\sim 8 \mathrm{GeV}^{2}$.

Our wave function provides the value

$$
\Gamma_{3 g}(J / \psi \rightarrow N \bar{N})=0.117 \mathrm{keV}
$$

for the three gluon contribution to the nucleonic $J / \psi$ width. If comparing the result with experiment 28] one has to be aware of the electromagnetic contribution and the spin effect. The electromagnetic contribution to $\mathcal{B}$ (see (5.3)) amounts to about $15 \%$ (see Sect. V). Since, however, the phase of the experimental time-like form factor is unknown we are not in the position to add $\mathcal{B}_{3 g}$ and $\mathcal{B}_{\text {em }}$ coherently at present. Thus, we can only say that, at best, the electromagnetic contribution may increase our prediction by $30 \%$. Considering also that $N \bar{N}$ pairs with equal helicity contribute about $10 \%$ to the total width, we regard our result for the three-gluon contribution as consistent with experiment.

Other sources of uncertainties in our calculation of the $J / \psi$ decay widths are introduced by the value of $f_{\Psi}$ chosen (see the discussion in Sect. 5) and by $\Lambda_{\mathrm{QCD}}$. Since $\alpha_{S}$ enters to the sixth power in $\Gamma_{3 g}$ the result (6.4) is rather sensitive to the value of $\Lambda_{\mathrm{QCD}}$ employed. A mild increase of $\Lambda_{\mathrm{QCD}}$ by $10 \%$ enlarges $\Gamma_{3 g}$ by about $30 \%$ without changing the re-

\footnotetext{
${ }^{7}$ In a common perturbative analysis of both the proton form factor and the $J / \Psi$ decay, the relative phase between the two contributions would be fixed.
} 
sults shown in Figs. 2 and 5 noticably; only the evolution behaviour of the nucleon wave function in the calculation of the Feynman contribution is slightly modified. Thus, we conclude that our wave function (2.11), (6.1) provides a reasonably large three gluon contribution to the $J / \psi \rightarrow p \bar{p}$ decay width. In contrast to previous calculations of this width carried through in collinear approximation, our average $\alpha_{S}$ (being 0.43 ) is consistent with the available scale in the $J / \psi$ decay which is provided by the $c$-quark mass. We also note that our perturbative calculation is self-consistent. The bulk of the contribution, i.e. more than $50 \%$, is accumulated in regions where $\alpha_{S}^{3}<0.47^{3}$.

At this point a remark concerning the $n \bar{n}$ decay channel is in order. Since the three-gluon contribution is flavour-blind any difference between the decay widths into $p \bar{p}$ and $n \bar{n}$ must be due to the electromagnetic contribution. From experiment it is known that the widths for $J / \psi \rightarrow p \bar{p}$ and $J / \psi \rightarrow n \bar{n}$ agree within the experimental errors 41 and that the time-like form factors for the proton and the neutron, to which the $\mathcal{B}_{\text {em }}$ are directly proportional, are approximately equal in modulus at $s=5.4 \mathrm{GeV}^{2}$ [42]. Since both the contributions, $\mathcal{B}_{3 g}$ and $\mathcal{B}_{\text {em }}$, are in general complex numbers with non-trivial phases the only conclusion to be drawn at present is that the relative phase between $\mathcal{B}_{3 g}$ and $\mathcal{B}_{\text {em }}$ is the same (up to an eventual sign) for the proton and the neutron channel.

The generalization to the decay $\Upsilon \rightarrow N \bar{N}$ is a straightforward task. For bottomium systems the application of our approach is even better justified than for charmonia because of the larger gluon virtualities. Using a value of $710 \mathrm{MeV}$ for the decay constant $f_{\Upsilon}$ of the $\Upsilon$ meson which, in a similar manner as $f_{\psi}$, is determined from the leptonic width of the $\Upsilon$ [28], we find $\Gamma_{3 g}(\Upsilon \rightarrow N \bar{N})=1.3 \cdot 10^{-2} \mathrm{eV}$. The electromagnetic contribution to $\Upsilon \rightarrow N \bar{N}$ decays is negligible as an estimate in analogy to that one in the $J / \Psi$ case shows. Up to now there is only an experimental upper limit of about $1 \mathrm{eV}$ for this decay width 28] which is safely met by our result. The effective $\alpha_{S}$ is 0.26 in the $\Upsilon$ case.

At the end of this section we want to comment on other forms of the nucleon's wave function to be found in the literature. For instance, $x$-independent parameterizations of $\Omega$ are used sometimes [2,23]. Although such factorizing forms of the wave functions 
are in conflict with rotational invariance and with the arguments given in Ref. [15], the numerical results, say, for the Feynman contribution to the nucleon form factor obtained with them do not differ much from our results. In other cases the wave function is regarded as a constituent wave function. Consequently the parameters are adjusted so that the wave function is normalized to unity and that the charge radius of the nucleon is reproduced. Constituent wave functions also provide large Feynman contributions which, however, decrease more rapidly with increasing momentum transfer than our results obtained from a wave function describing an object that is smaller than the nucleon. The introduction of form factors for the constituent quarks mediating the transition to current quarks, may improve the large momentum transfer behaviour of the form factor (see, for instance, Ref. 43]). Another possibility to construct a wave function is to start from an equal time wave function (e. g. that of a harmonic oscillator) in the nucleon's rest frame and transform it to the light cone under the assumption that a Melosh transform for free, non-interacting quarks can be applied (see, for instance, Ref. [44]). While this is a very interesting approach attempting to construct a unified picture of the non-relativistic quark model and light-cone physics, the resulting wave function presented in Ref. [44] does not pass our tests against data.

Occasionally DAs are used which possess a multiplicative factor $\exp \left[-a^{2} \sum_{i} m_{q}^{2} / x_{i}\right]$ (see, for instance, Refs. [14,44) where the parameter $m_{q}$ is to be interpreted as a constituent quark mass. Although the infinite series (2.7) can, in principle, accommodate such a mass exponential, the truncated expansion may not reproduce it sufficiently accurate. It may perhaps be better to consider the mass exponential explicitly. To get an idea about the importance of that mass exponential we modify the DA (6.1) by it and repeat our calculations. It turns out that the $J / \psi$ decay width as well as the distribution functions are mildly affected by the mass exponential while the Feynman contribution is reduced by about $20 \%$ in the momentum transfer region between 5 and $15 \mathrm{GeV}^{2}$ but decreases somewhat faster with increasing $Q^{2}$ than the Feynman contribution obtained from the original DA (6.1). The reduction around $10 \mathrm{GeV}^{2}$ can be compensated for by a $10 \%$ 
increase of $f_{N}$.

Finally, one may think of additional powers of transverse momenta multiplying the Gaussian (2.11) (see, for instance, Ref. [44]). Despite of this and many other possible complications we stick to our simple ansatz (2.1), (2.5) and (2.11) because, as we have shown, it is flexible enough to account for the available data with a sufficient degree of accuracy.

\section{SUMMARY AND CONCLUSIONS}

In this paper we have investigated the soft light-cone wave function of the nucleon. For the DAs we use the expansion in terms of the eigenfunctions of the evolution equation truncated at some finite order. For the transverse momentum dependence of the wave function we use a specific form which is supported by results obtained in 14, 15. On the strength of rather general arguments the $k_{\perp}$-dependence of the wave function appears in the form $k_{\perp i}^{2} / x_{i}$ with a Gaussian fall-off at large $k_{\perp}$. Studying soft Feynman contributions to the Dirac form factor $F_{1}^{N}$ of the nucleon and valence Fock state contributions to the quark distribution functions of the nucleon at large $x$ we found that wave functions constructed on the basis of QCD sum rules in general lead to too large predictions. These results resemble similar considerations in the case of the pion [\&]. In the pion case there is strong evidence that the DA is close to the asymptotic one, whereas in the case at hand asymmetries between the proton and neutron form factor as well as structure function data prevent the use of the asymptotic DA.

We take these observations as a motivation to model a new wave function which consistently describes the valence Fock state contributions to the quark distribution functions

and the Dirac form factor $F_{1}^{N}$ by its Feynman contribution. In addition we require that it also leads to a proper prediction for the $J / \psi \rightarrow N \bar{N}$ decay width within the modified perturbative approach. The perturbative calculation is self-consistent in that case and, in contrast to the case of the nucleon form factor, the perturbative contribution is in fair agreement with the experimental result on the decay width. It should be noted that 
there is still some residual uncertainty in the perturbative contribution from the imperfect knowledge of the strong coupling constant $\alpha_{S}$ and the $J / \psi$ decay constant. At any rate we have been able to rectify the treatment of $\alpha_{S}$ by avoiding some fixed scale prescriptions in contrast to previous calculations. In our calculation the effective value of $\alpha_{S}$ is 0.43 for our wave function at $\Lambda_{\mathrm{QCD}}=220 \mathrm{MeV}$ instead of about 0.3 as in previous calculations. The wave function which we determine from the combined fit to the three sets of data consists of a Gaussian $k_{\perp}$-dependence and a very simple DA which bears resemblance to the asymptotic DA in shape but with the position of the only maximum shifted somewhat. Like the COZ-type DAs our DA possesses the interesting property that, on the average, a $u$-quark in the proton carries a larger fraction of the proton's momentum than the $d$-quark. Our wave function defined by (2.1), (2.5), (2.11) and (6.1), has only two free parameters to be adjusted. With more complicated wave functions, containing more free parameters, the fit can certainly be improved. However, with regard to our aim of demonstrating the existence of a soft nucleon wave function which complies with theoretical ideas and from which the prominent features of the data can be reproduced, we refrain from introducing such complications.

\section{ACKNOWLEDGMENTS}

We would like to thank N.G. Stefanis for useful discussions and S.J. Brodsky for valuable comments.

\section{APPENDIX A: PERTURBATIVE CALCULATION OF THE $J / \psi \rightarrow N \bar{N}$ DECAY AMPLITUDE}

As in previous perturbative calculations [10 13] the $J / \psi$ meson will be treated as a non-relativistic $c \bar{c}$ system with $v^{2} / c^{2}$ corrections neglected. According to 450 we write the 
$J / \psi$ state in a covariant fashion

$$
|J / \psi ; q, \lambda\rangle=\frac{\delta_{a b}}{\sqrt{3}} \frac{f_{\psi}}{2 \sqrt{6}} \frac{\left(\not t+M_{\psi}\right) \notin(\lambda)}{\sqrt{2}},
$$

where $a$ and $b$ are color indices. Within the modified perturbative approach the threegluon contribution $\mathcal{B}_{3 g}$ to the $J / \psi$ decay into $N \bar{N}$ is of the form

$$
\begin{aligned}
& \mathcal{B}_{3 g}=\frac{f_{\psi}}{2 \sqrt{6}} \int[\mathrm{d} x]\left[\mathrm{d} x^{\prime}\right] \int \frac{\mathrm{d}^{2} \mathbf{b}_{1}}{(4 \pi)^{2}} \frac{\mathrm{d}^{2} \mathbf{b}_{3}}{(4 \pi)^{2}} \hat{T}_{H}\left(x, x^{\prime}, \mathbf{b}\right) \exp \left[-S\left(x, x^{\prime}, M_{\psi}\right)\right] \\
& \times\left[\hat{\Psi}_{123}(x, \mathbf{b}) \hat{\Psi}_{123}\left(x^{\prime}, \mathbf{b}\right)+\frac{1}{2}\left(\hat{\Psi}_{123}(x, \mathbf{b})+\hat{\Psi}_{321}(x, \mathbf{b})\right)\left(\hat{\Psi}_{123}\left(x^{\prime}, \mathbf{b}\right)+\hat{\Psi}_{321}\left(x^{\prime}, \mathbf{b}\right)\right)\right] .
\end{aligned}
$$

This convolution of wave functions and a hard scattering amplitude $T_{H}$ can formally be derived by using the methods described in detail by Botts and Sterman [3]. The $\mathbf{b}_{i}$, canonically conjugated to the transverse momenta $\mathbf{k}_{\perp i}$, are the quark separations in the transverse configuration space. $\mathbf{b}_{1}$ and $\mathbf{b}_{3}$ correspond to the locations of quarks 1 and 3 in the transverse plane relative to quark 2 and $\mathbf{b}_{2}=\mathbf{b}_{1}-\mathbf{b}_{3} . \hat{\Psi}_{i j k}$ represents the Fourier transform of the wave function $\Psi_{i j k}$.

The hard scattering amplitude, to be calculated from Feynman graphs of the type shown in Fig. 83, reads

$$
T_{H}\left(x, x^{\prime}, \mathbf{k}_{\perp}, \mathbf{k}_{\perp}^{\prime}\right)=\frac{5120 \sqrt{6} / 27 \pi^{3} M_{\psi}^{5}\left(x_{1} x_{3}^{\prime}+x_{3} x_{1}^{\prime}\right)}{\left[\tilde{q}_{1}^{2}+\left(\mathbf{k}_{\perp 1}+\mathbf{k}_{\perp 1}^{\prime}\right)^{2}\right]\left[\tilde{q}_{3}^{2}+\left(\mathbf{k}_{\perp 3}+\mathbf{k}_{\perp 3}^{\prime}\right)^{2}\right]} \prod_{i=1}^{3} \frac{\alpha_{S}\left(t_{i}\right)}{\tilde{g}_{i}^{2}-\left(\mathbf{k}_{\perp i}+\mathbf{k}_{\perp i}^{\prime}\right)^{2}+i \epsilon},
$$

where

$$
\tilde{q}_{i}^{2}=\left[x_{i}\left(1-x_{i}^{\prime}\right)+\left(1-x_{i}\right) x_{i}^{\prime}\right] M_{\psi}^{2} / 2, \quad \tilde{g}_{i}^{2}=x_{i} x_{i}^{\prime} M_{\psi}^{2} .
$$

Note that $T_{H}$ depends on sums of transverse momenta, $\mathbf{K}_{i} \equiv \mathbf{k}_{\perp i}+\mathbf{k}_{\perp i}^{\prime}$, and because of the constraint $\sum_{i} \mathbf{K}_{i}=\mathbf{0}$ only two of the $\mathbf{K}_{i}$, say $\mathbf{K}_{1}$ and $\mathbf{K}_{3}$, are independent. Hence, the Fourier transformed hard amplitude

$$
\hat{T}_{H}\left(x, x^{\prime}, \mathbf{b}_{1}, \mathbf{b}_{3}\right)=\int \frac{\mathrm{d}^{2} \mathbf{K}_{1}}{(2 \pi)^{2}} \frac{\mathrm{d}^{2} \mathbf{K}_{3}}{(2 \pi)^{2}} T_{H}\left(x, x^{\prime}, \mathbf{K}\right) \exp \left[-i \mathbf{K}_{1} \cdot \mathbf{b}_{1}-i \mathbf{K}_{3} \cdot \mathbf{b}_{3}\right],
$$

depends only on the vectorial distances $\mathbf{b}_{1}$ and $\mathbf{b}_{3}$. In physical terms this means that the $N \bar{N}$ pairs emerge with identical transverse separation configurations from the decay 
because each gluon produces a quark-antiquark pair at the same location in the transverse configuration plane which thereafter do not interact. We emphasize that in contrast to the case of the nucleon form factor [4] this circumstance is not due to approximations concerning the $k_{\perp}$-dependences of $T_{H}$ but instead a direct consequence of the decay kinematics.

Inserting (A3) into (A5), one finds for the hard scattering amplitude in $\mathbf{b}$ space

$$
\begin{aligned}
\hat{T}_{H}\left(x, x^{\prime}, \mathbf{b}_{1}, \mathbf{b}_{3}\right) & =-\frac{2560}{27} f_{\psi} M_{\psi}^{5} \frac{\left(x_{1} x_{3}^{\prime}+x_{3} x_{1}^{\prime}\right)}{\left[\tilde{q}_{1}^{2}+\tilde{g}_{1}^{2}\right]\left[\tilde{q}_{3}^{2}+\tilde{g}_{3}^{2}\right]} \frac{1}{(2 \pi)^{3}} \prod_{i=1}^{3}\left(\pi \alpha_{S}\left(t_{i}\right)\right) \int \mathrm{d}^{2} \mathbf{b}_{0} \\
& \times\left[\frac{i \pi}{2} \mathrm{H}_{0}^{(1)}\left(\tilde{g}_{1}\left|\mathbf{b}_{1}+\mathbf{b}_{0}\right|\right)-\mathrm{K}_{0}\left(\tilde{q}_{1}\left|\mathbf{b}_{1}+\mathbf{b}_{0}\right|\right)\right] \frac{i \pi}{2} \mathrm{H}_{0}^{(1)}\left(\tilde{g}_{2} b_{0}\right) \\
& \times\left[\frac{i \pi}{2} \mathrm{H}_{0}^{(1)}\left(\tilde{g}_{3}\left|\mathbf{b}_{3}+\mathbf{b}_{0}\right|\right)-\mathrm{K}_{0}\left(\tilde{q}_{3}\left|\mathbf{b}_{3}+\mathbf{b}_{0}\right|\right)\right]
\end{aligned}
$$

The auxiliary variable $\mathbf{b}_{0}$ in Eq. (A6) serves as a Lagrange multiplier to the constraint $\sum \mathbf{K}_{i}=\mathbf{0}$. Inserting (A6) into the expression (A2) for the invariant function $\mathcal{B}_{3 g}$ we see that a nine dimensional numerical integration is to be performed. Although this is a rather involved technical task it can be carried through with sufficient accuracy if some care is put into it. Since the virtualities of the gluons are timelike, $\hat{T}_{H}$ includes complexvalued Hankel functions $\mathrm{H}_{0}^{(1)}$ which are related to the usual modified Bessel functions $\mathrm{K}_{0}$, appearing for space-like propagators, by analytic continuation in the momentum transfer variable which in our case is the $J / \psi$ mass. Thus, $\mathcal{B}_{3 g}$ has a non-trivial phase as for instance the time-like form factors [46].

The Sudakov factor $\exp [-S]$ in (A2) takes into account those gluonic radiative corrections not accounted for in the QCD evolution of the wave function as well as the renormalization group transformation from the factorization scale $\mu_{F}$ to the renormalization scales $t_{i}$ at

\footnotetext{
${ }^{8}$ Taking into account relativistic corrections to the $J / \psi$ wave function, i.e. its transverse momentum dependence, one would have to perform a 14 dimensional numerical integration which is impossible with present day computers to a sufficient degree of accuracy.
} 
which the hard amplitude $\hat{T}_{H}$ is evaluated. The Sudakov exponent reads

$$
S\left(x, x^{\prime}, M_{\psi}\right)=\sum_{i=1}^{3}\left[s\left(x_{i}, \tilde{b}, M_{\psi}\right)+s\left(x_{i}^{\prime}, \tilde{b}, M_{\psi}\right)+\frac{4}{\beta} \log \frac{\log \left(t_{i} \Lambda_{\mathrm{QCD}}\right)}{\log \left(1 / \tilde{b}_{i} \Lambda_{\mathrm{QCD}}\right)}\right] \text {, }
$$

where $\beta \equiv 11-2 / 3 n_{f}$. The function $s\left(\xi, \tilde{b}, M_{\psi}\right)$, originally derived by Botts and Sterman [3] and later on slightly improved, can be found in Refs. [5,47]. $M_{\psi}$ appears in the function $s$ since it provides the large scale in the process of interest.

The quantities $\tilde{b}_{i}$ are infrared cut-off parameters, naturally related to, but not uniquely determined by the mutual separations of the three quarks [48]. Following [4] we chose $\tilde{b}_{i}=\tilde{b}=\max \left\{b_{1}, b_{2}, b_{3}\right\}$. With this "MAX" prescription the hard scattering amplitude is unencumbered by $\alpha_{S}$ singularities in the soft end-point regions. As a consequence of the regularizing power of the "MAX" prescription, the perturbative contribution saturates in the sense that the results become insensitive to the inclusion of the soft regions. A saturation as strong as possible is a prerequisite for the self-consistency of the perturbative approach. The infrared cut-off $\tilde{b}$ marks the interface betweeen the non-perturbative soft gluons, which are implicitly accounted for in the nucleon wave function, and the contributions from soft gluons, incorporated in a perturbative way in the Sudakov factor. Obviously, the infrared cut-off serves at the same time as the gliding factorization scale $\mu_{F}$ to be used in the evolution of the wave function.

The renormalization scales $t_{i}$ are defined in analogy to the case of electromagnetic form factors [3,4] as the maximum scale of either the longitudinal momentum or the inverse transverse separation associated with each of the gluons

$$
t_{1}=\max \left(\tilde{q}_{1}, \tilde{g}_{1}, 1 / b_{3}\right), \quad t_{2}=\max \left(\tilde{g}_{2}, 1 / b_{2}\right), \quad t_{3}=\max \left(\tilde{q}_{3}, \tilde{g}_{3}, 1 / b_{1}\right)
$$

The above assignment of $b$-scales is not compelling. Rearrangements in the $b$-scales, however, induce only slight changes in the numerical results. 


\section{REFERENCES}

[1] G.P. Lepage, S.J. Brodsky: Phys. Rev. D22 (1980) 2157.

[2] N. Isgur, C.H. Llewellyn-Smith: Nucl. Phys. B317 (1989) 526.

[3] J. Botts, G. Sterman: Nucl. Phys. B325 (1989) 62;

H.-N. Li, G. Sterman: Nucl. Phys. B381 (1992) 129.

[4] J. Bolz, R. Jakob, P. Kroll, M. Bergmann, N.G. Stefanis: Z. Phys. C66 (1995) 267;

Phys. Lett. B342 (1995) 345.

[5] J. Bolz: Ph.D. thesis, WUB - DIS 95-10, University of Wuppertal, 1995; work in preparation.

[6] V.L. Chernyak, A.A. Ogloblin, I.R. Zhitnitsky: Z. Phys. C42 (1989) 569.

[7] A.F. Sill et al. : Phys. Rev. D48 (1993) 29.

[8] R. Jakob, P. Kroll, M. Raulfs: J. Phys. G22 (1996) 45.

[9] M. Glück, E. Reya, A. Vogt: Z. Phys. C67 (1995) 433.

[10] S.J. Brodsky, G.P. Lepage: Phys. Rev. D24 (1981) 2848.

[11] V.L. Chernyak, A.A. Ogloblin, I.R. Zhitnitsky: Z. Phys. C42 (1989) 583.

[12] N.G. Stefanis, M. Bergmann: Phys. Rev. D47 (1993) 3685.

[13] P.H. Damgaard, K. Tsokos, E.L. Berger: Nucl. Phys. B259 (1985) 285.

[14] G.P. Lepage, S.J. Brodsky, T. Huang, P.B. Mackenzie: Banff Summer Institute, Particles and Fields 2, A.Z. Capri, A.N. Kamal (eds.) (1983), p. 83;

S.J. Brodsky, T. Huang, G.P. Lepage: ibid. , p. 143.

[15] B. Chibisov, A.R. Zhitnitsky: Phys. Rev. D52 (1995) 5273.

[16] M.G. Sotiropoulos, G. Sterman: Nucl. Phys. B425 (1994) 489. 
[17] Z. Dziembowski: Phys. Rev. D37 (1988) 768.

[18] M. Peskin: Phys. Lett. B88 (1979) 128.

[19] N.G. Stefanis, M. Bergmann: Phys. Rev. D48 (1993) 2990;

Phys. Lett. B325 (1994) 183.

[20] S.D. Drell, T.-M. Yan: Phys. Rev. Lett. 24 (1970) 181.

[21] G.B. West: Phys. Rev. Lett. 24 (1970) 1206.

[22] S.D. Drell, D.J. Levy, T.M. Yan: Phys. Rev. 187 (1969) 2159.

[23] C.E. Carlson, F. Gross: Phys. Rev. D36 (1987) 2060.

[24] A. Schäfer, L. Mankiewicz, Z. Dziembowski: Phys. Lett. B233 (1989) 217.

[25] M.L. Mangano, A. Petrelli: Phys. Lett. B352 (1995) 445.

[26] M. Consoli, J.M. Field: Phys. Rev. D49 (1994) 1293.

[27] D. Pallin et al. (DM2 collaboration): Nucl. Phys. B292 (1987) 653.

[28] Particle Data Group: Review of Particle Properties, Phys. Rev. D50 (1994) 1173.

[29] R. Barbieri, R. Kögerler, Z. Kunszt, R. Gatto: Nucl. Phys. B105 (1976) 125.

[30] T. A. Armstrong et al. (E760 collaboration): Phys. Rev. Lett. 70 (1993) 1212.

[31] M. Gari, N.G. Stefanis: Phys. Lett. B175 (1986) 462.

[32] G. Martinelli, C.T. Sachrajda: Phys. Lett. B217 (1989) 319.

[33] K.C. Bowler et al. : Nucl. Phys. B296 (1988) 431.

[34] S.J. Brodsky, J. Ellis, J.S. Hagelin, C.T. Sachrajda: Nucl. Phys. B238 (1984) 561.

[35] P. Langacker: Preprint Penn University UPR-0263T, published in proceedings of the workshop "Inner space/ outer space", Batavia, IL, May 2-5, 1984, edts. E.W. Kolb, 
M.S. Turner, D. Lindley, K.A. Olive and D. Seckel, University of Chicago Press (1986).

[36] V.L. Chernyak, A.R. Zhitnitsky: Phys. Rep. 112 (1984) 173.

[37] A. Duncan, S. Pernice, E. Schnapka: "Pion wave function and truncation sensitivity of QCD sum rules", hep-ph/9602247.

[38] N. Isgur, G. Karl, R. Koniuk: Phys. Rev. Lett. 41 (1978) 1269; ibid. 45 (1980) 1738(E).

[39] A. Lung et al. : Phys. Rev. Lett. 70 (1993) 718.

[40] A.R. Zhitnitsky: Preprint SMU Dallas, SMU-HEP-94-01 (1994), hep-ph/9402280.

[41] A. Antonelli et al. (FENICE): Phys. Lett. B301 (1993) 317.

[42] A. Antonelli et al. (FENICE): Phys. Lett. B313 (1993) 283.

[43] F. Cardelli, E. Pace, G. Salmè, S. Simula: Phys. Lett. B357 (1995) 267.

[44] Z. Dziembowski: in Quark Cluster Dynamics, Lecture Notes in Physics, Vol. 417, edited by K. Goeke, P. Kroll and H. R. Petry (Springer Verlag, Berlin, Heidelberg (1992); Z. Dziembowski, C. J. Martoff, P. Żyła: Phys. Rev. D50 (1994) 5613.

[45] J.H. Kühn, J. Kaplan, E.G.O. Safiani: Nucl. Phys. B157 (1979) 125.

[46] T. Gousset, B. Pire: Phys. Rev. D51 (1995) 15.

[47] M. Dahm, R. Jakob, P. Kroll: Z. Phys. C68 (1995) 595.

[48] J.C. Collins, D.E. Soper: Nucl. Phys. B193 (1981) 381. 


\section{FIGURES}

FIG. 1. Feynman contributions to Dirac form factor $F_{1}^{p}$ using the COZ wave function. The solid (dashed, dotted) line is evaluated with $a=0.99(0.60,0.45) \mathrm{GeV}^{-1}$. Experimental data (०) are taken from [7].

FIG. 2. Valence Fock state contributions to the valence quark distribution functions of the proton at $Q^{2}=1 \mathrm{GeV}^{2}$. The open circles represent the parameterization of Ref. 91. The solid and dashed lines represent the contributions of the valence Fock state using a wave function composed of the Gaussian (2.11) and either the DA (6.1) or the COZ one $\left(a=0.60 \mathrm{GeV}^{-1}\right)$, respectively. The dotted line is obtained from the AS wave function with $f_{N}$ and $a$ as for the wave function (2.11), 6.1).

FIG. 3. Decay graph $J / \psi \rightarrow 3 g \rightarrow 3 q \bar{q}$. The momenta of the quarks are $x_{i} p+k_{i}$ with $k_{i}=\left(0,0, \mathbf{k}_{\perp i}\right)$, and those of the antiquarks are marked by a prime.

FIG. 4. The DA (6.1) as a function of $x_{1}$ and $x_{3}$.

FIG. 5. Feynman contribution to the Dirac form factor of the proton (top) and the neutron (bottom) evaluated from the wave function (2.11), (6.1). The dashed line in the upper figure is obtained from the AS wave function with $f_{N}$ and $a$ as for the wave function (2.11), (6.1). Data (०) are taken from Refs. [7,39]. 


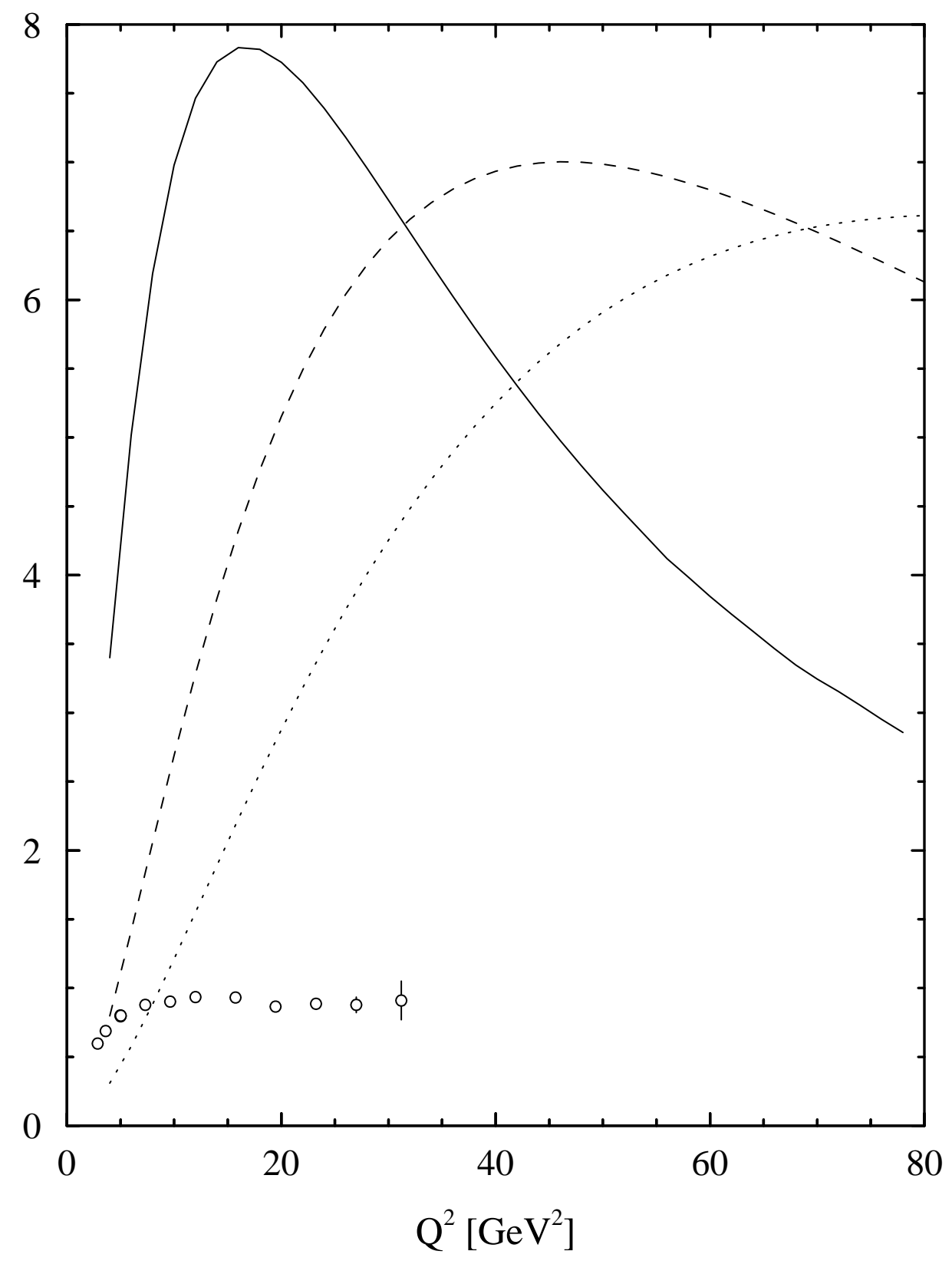

Figure 1 

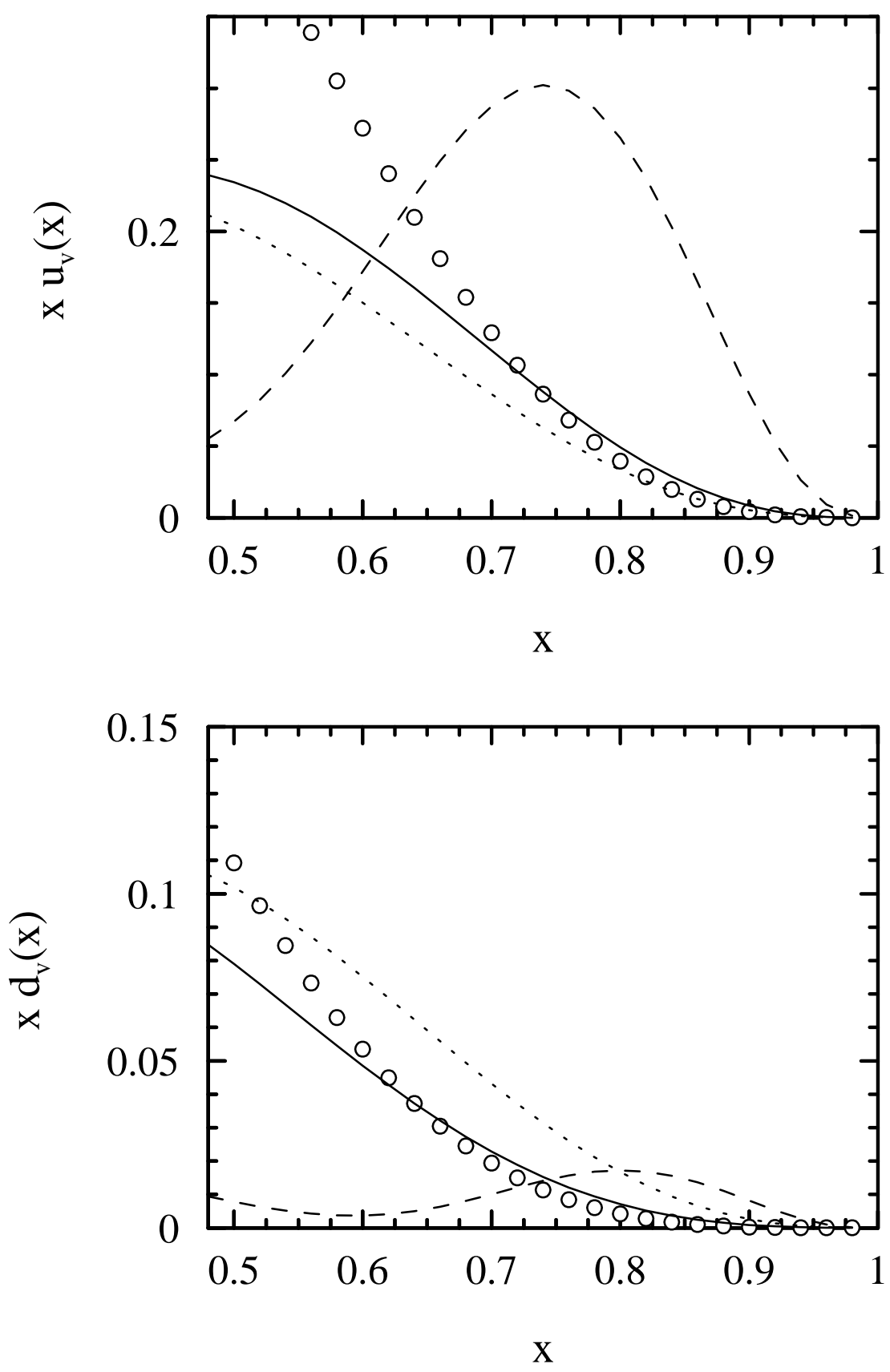

Figure 2 


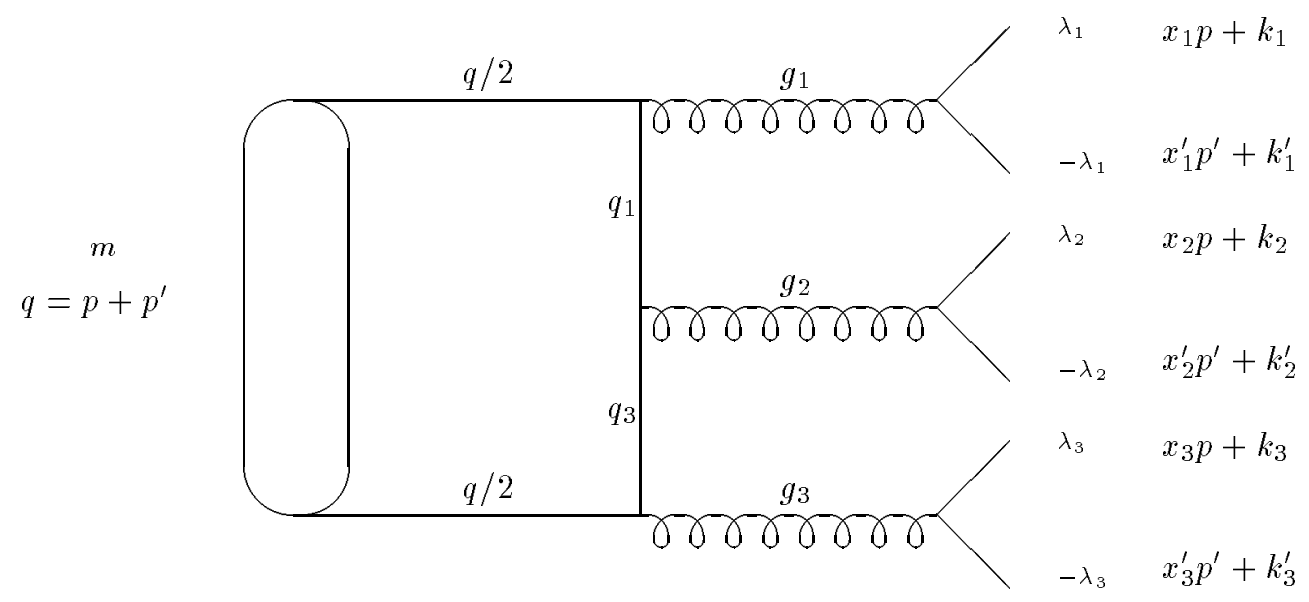

Figure 3

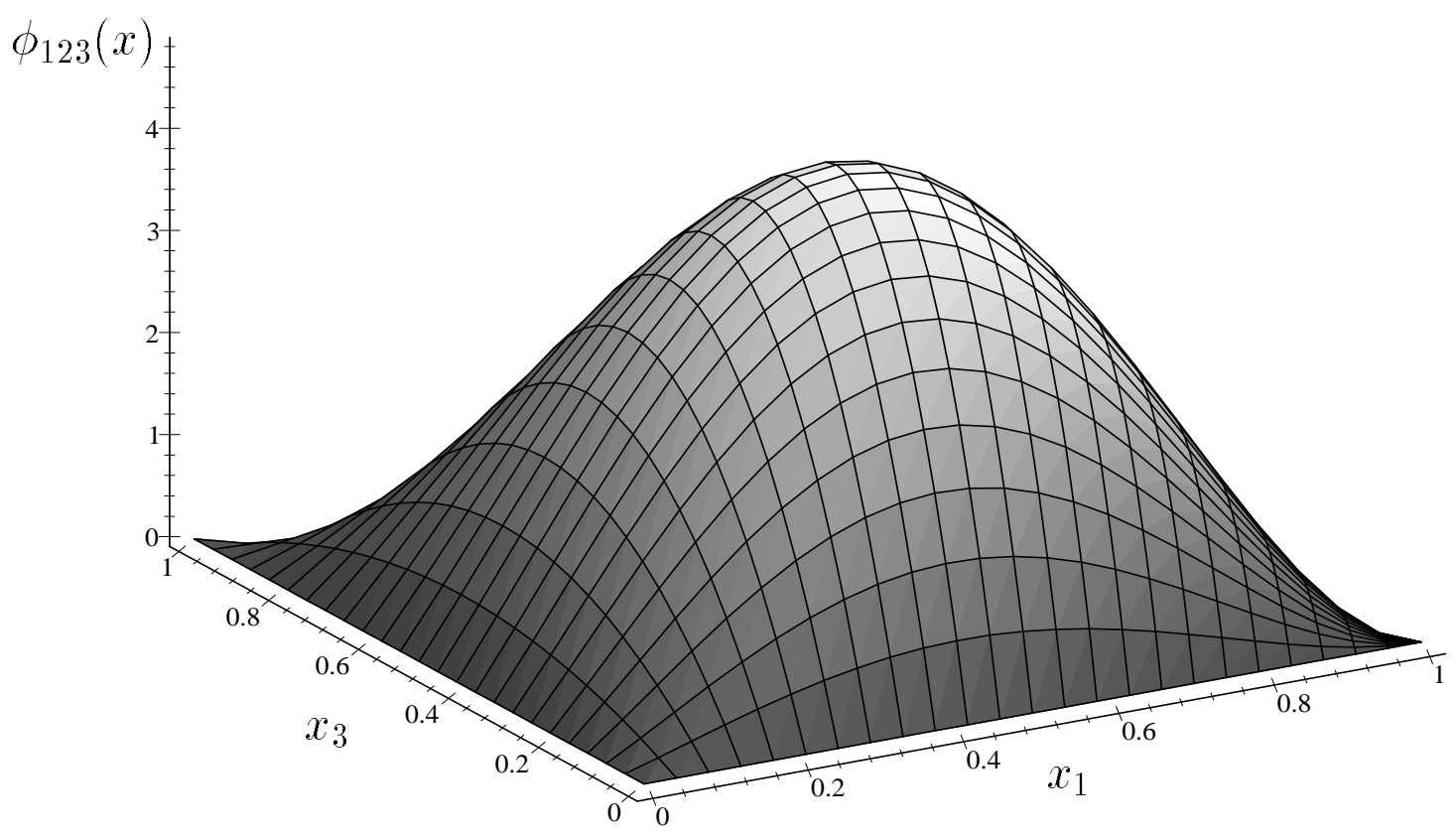

Figure 4 

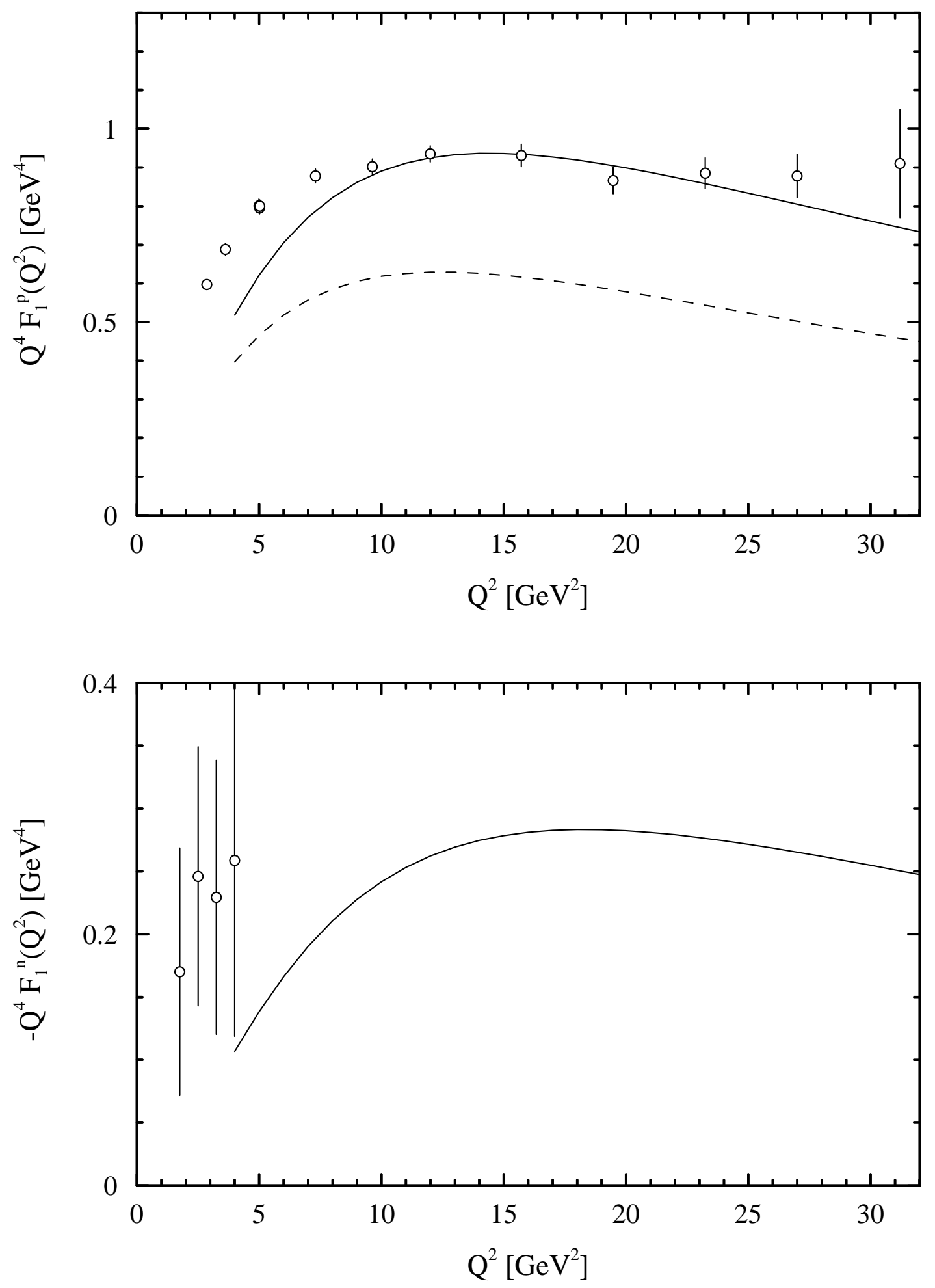

Figure 5 